(1)APO

\title{
Protocolo de terapia fotodinâmica e fotobiomodulação no tratamento de herpes simples labial-fase vesicular: relato de dois casos clínicos
}

\author{
Photobiomodulation and photodynamic therapy protocol on the treatment of \\ herpes simplex labialis - vesicle phase: report of two clinical cases
}

\author{
Roberta Souza D'Almeida Couto ${ }^{1}$ \\ Luciane Hiramatsu Azevedo ${ }^{2}$ \\ Thais Mendonça Petta ${ }^{3}$ \\ Tamea Lacerda Monteiro Medeiros ${ }^{3}$ \\ Patrícia Moreira de Freitas ${ }^{2}$
}

\section{RESUMO}

O Herpes simples labial é uma doença contagiosa causada pelo vírus do herpes simples tipo 1 (HSV-1). O vírus é capaz de residir por toda a vida no hospedeiro infectado, apresentando períodos de latência e reativação com disseminação. O HSV-1 dissemina-se predominantemente através da saliva infectada ou de lesões periorais ativas. O tratamento convencional é por medicamentos antivirais e sua ação é na fase prodrômica aliviando coceira e dor. $\mathrm{O}$ uso intermitente das medicações não altera a frequência da recorrência e ainda tem sido associado com o aparecimento de vírus resistentes aos medicamentos, especialmente em pacientes imunocomprometidos. O objetivo do trabalho foi relatar um protocolo de tratamento para herpes simples labial na fase vesicular utilizando as terapias fotodinâmica (TFD) e fotobiomodulação (FBM), como alternativa de tratamento. Dois casos clínicos foram realizados no Laboratório Especial de Laser em Odontologia (LELO) da Faculdade de Odontologia da Universidade de São Paulo/USP. A TFD foi realizada nos pacientes e, após 24h, a terapia de fotobiomodulação (FBM) com laser de baixa potência. Os resultados mostraram sucesso nos casos clínicos e na fotoinativação do vírus pela TFD. O tratamento com laser de baixa potência, tanto para a TFD como para FBM foi efetivo e seguro, resultando em descontaminação e cicatrização da área da lesão afetada, além da melhora dos sinais e sintomas do herpes simples labial.

Palavras chave: Herpes simples labial. Terapia fotodinâmica. Terapia a laser de baixa potência.

\begin{abstract}
Herpes simplex labialis is a contagious disease caused by the herpes simplex virus type 1 (HSV-1). The virus is capable of living throughout the life of the infected host, presenting periods of latency and reactivation with propagation. The HSV-1 diffuses predominantly through infected saliva or active perioral lesions. The conventional treatment is given by antiviral medication and its action is in the prodromic phase relieving itching and pain. The intermittent usage of medications does not alter the frequency of recurrence and it has been associated with the arising of $\mathrm{t}$ viruses that are resistant to medication, especially in immunocompromised patients. The objective of the study was to report a treatment protocol for herpes simplex labialis in the vesicular phase using photodynamic therapy (PDT) and photobiomodulation (PBM) therapy as an alternative treatment. Two clinical cases were performed at the Special Laser Laboratory of Dentistry (LELO) of the School of Dentistry of the University of São Paulo / USP. Patients received PDT and, after $24 \mathrm{~h}$, the photobiomodulation (PBM) therapy with low intensity laser. The results showed success on the clinical cases and photoinactivation of the virus by PDT. The treatment with low intensity laser, for both PDT and PBM, was effective and safe, resulting in decontamination and healing of the area of the affected lesion, and also the improvement of the signs and symptoms of herpes simplex labialis.
\end{abstract}

Keyword: Herpes simplex labialis. Photodynamic therapy. Low-level laser therapy.

\footnotetext{
${ }^{1}$ Doutora, Universidade Federal do Pará

${ }^{2}$ Doutora, Universidade de São Paulo

${ }^{3}$ Mestranda, Universidade Federal do Pará
} 


\section{INTRODUÇÃo}

O Herpes simples labial é uma doença contagiosa causada pelo vírus do herpes simples tipo 1 (HSV-1). O vírus é capaz de residir por toda a vida no hospedeiro infectado, apresentando períodos de latência e reativação com disseminação. O HSV-1 dissemina-se predominantemente através da saliva infectada ou de lesões periorais ativas. As lesões labiais clássicas envolvem o desenvolvimento sequencial de eritema, pápulas, vesículas, pústulas, úlceras e crosta. $\mathrm{O}$ pico viral é em 24 h após a formação da lesão, na fase de vesícula. Os pacientes geralmente apresentam o estágio prodrômico de dor, queimação, coceira e parestesia, o que pode prejudicar a qualidade de vida do indivíduo, especialmente na fase de vesícula ${ }^{1,2}$. O tratamento convencional é por medicamentos antivirais e sua ação é na fase prodrômica aliviando coceira e dor. O uso intermitente das medicações não altera a frequência da recorrência e ainda tem sido associado com o aparecimento de vírus resistentes aos medicamentos, especialmente em pacientes imunocomprometidos e idosos ${ }^{3}$.

O recente aumento da resistência aos antibióticos em todo o mundo tem renovado o interesse em terapias antimicrobianas alternativas. A terapia fotodinâmica (TFD) é uma técnica baseada na presença de espécies reativas de oxigênio (EROs) produzidas por moléculas de fotossensibilização na presença de luz visível em baixa intensidade. São produzidos superóxidos, oxigênios simples, radicais hidroxílicos que são citotóxicos às células microbianas e às células do hospedeiro ${ }^{4,5}$. A escolha da terapia deve ser feita de acordo com o estágio da lesão do herpes. Quando a lesão está na fase vesicular, a TFD deve ser usada. Quando as crostas já estão formadas, a terapia de fotobiomodulação (FBM) com laser de baixa potência, com emissão no espectro do vermelho, pode ser usado com o objetivo de acelerar o processo de cicatrização ${ }^{6}$.

Assim, o objetivo do presente trabalho foi relatar o protocolo de tratamento de dois casos clínicos para herpes simples labial na fase vesicular utilizando a terapia fotodinâmica (TFD) associada à fotobiomodulação como alternativa de tratamento.

\section{RELATO DO CASO CLÍNICO}

Os dois casos clínicos apresentados foram realizados no Laboratório Especial de Laser em Odontologia (LELO) do Departamento de Dentística da Faculdade de Odontologia da Universidade de São Paulo/USP. Os pacientes atendidos assinaram o termo de consentimento informado para o tratamento de herpes simples labial. Os pacientes apresentavam o herpes na fase vesicular e, portanto, a indicação foi de realizar o tratamento de terapia fotodinâmica (TFD) nas lesões. A Tabela 01 mostra os parâmetros de irradiação do laser para TFD. A Tabela 02 mostra os parâmetros de irradiação do laser para a terapia de fotobiomodulação (FBM). O protocolo de tratamento para herpes simples labial (fase vesicular) utilizado nos casos clínicos 1 e 2 são descritos a seguir:

Tabela 01. Parâmetros da dosimetria utilizado na Terapia Fotodinâmica (TFD).

\begin{tabular}{|l|r|}
\hline \multicolumn{2}{|c|}{ Parâmetros de irradiação TFD } \\
\hline Comprimento de onda (nm) & 660 \\
\hline Meio ativo & InGaAlP \\
\hline Área do feixe $\left(\mathbf{c m}^{\mathbf{2}}\right)$ & 0,04 \\
\hline Potência $(\mathbf{m W})$ & 40 \\
\hline Densidade de Potência $\left(\mathbf{W} / \mathbf{c m}^{\mathbf{2}}\right)$ & 120 \\
\hline Densidade de energia $\left(\mathbf{J} / \mathrm{cm}^{\mathbf{2}}\right)$ & 120 \\
\hline Tempo de irradiação por ponto (s) & 4,8 \\
\hline Energia por ponto $(\mathbf{J})$ & Pontual e contato \\
\hline Modo de irradiação & 1 \\
\hline Número de sessões & 120 \\
\hline
\end{tabular}

Tabela 02. Parâmetros da dosimetria utilizado na Terapia de Fotobiomodulação (FBM).

\begin{tabular}{|l|r|}
\hline \multicolumn{2}{|c|}{ Parâmetros de irradiação TC } \\
\hline Comprimento de onda $(\mathbf{n m})$ & 660 \\
\hline Meio ativo & InGaAlP \\
\hline Área do feixe $\left(\mathbf{c m}^{\mathbf{2}}\right)$ & 0,04 \\
\hline Potência $(\mathbf{m W})$ & 15 \\
\hline Densidade de Potência $\left(\mathbf{W} / \mathbf{c m}^{\mathbf{2}}\right)$ & 0,375 \\
\hline Densidade de energia $\left(\mathbf{J} / \mathbf{c m}^{\mathbf{2}}\right)$ & 3,7 \\
\hline Tempo de irradiação por $\mathbf{~ p o n t o ~}(\mathbf{s})$ & 10 \\
\hline Energia por ponto $(\mathbf{J})$ & 0,15 \\
\hline Modo de irradiação & 1 a 2 \\
\hline Número de sessões & 24 \\
\hline Intervalo entre as sessões $(\mathbf{h})$ & Pontual e contato \\
\hline
\end{tabular}




\section{Caso clínico 1}

Paciente RWP, sexo masculino, 23 anos, fototipo de pele parda, apresentou vesículas no lábio inferior esquerdo (Fig.1A), com sintomatologia dolorosa e em queimação no local da lesão. As vesículas foram cuidadosamente perfuradas com agulha estéril (Fig.1B) e o fluido drenado cuidadosamente com auxílio de gaze estéril para evitar espalhar infecção para área adjacente. Em seguida, foi aplicado o fotossensibilizador azul de metileno 0,005\% (Chimiolux®, DMC, São Carlos, SP, BR) sobre a lesão (Fig.1C) e, após 5 min, o excesso de fotossensibilizador foi removido. A área da lesão foi irradiada em 3 pontos distintos, utilizando um equipamento de laser de diodo, emissão contínua, em baixa intensidade com comprimento de onda de $660 \mathrm{~nm}$ (Twin Laser; MM Optics®, São Carlos, SP, BR), potência de saída de $40 \mathrm{mw}$, densidade de energia de 120 $\mathrm{J} / \mathrm{cm}^{2}, 2$ min por ponto, no modo contato (Fig.1D). Após $24 \mathrm{~h}$, o paciente iniciou a terapia de fotobiomodulação (FBM). Para tanto, a área da lesão foi irradiada em 6 pontos distintos com o mesmo equipamento de laser de baixa potência, com comprimento de onda de $660 \mathrm{~nm}$, porém com potência de saída de $15 \mathrm{mw}$, densidade de energia de $3,7 \mathrm{~J} / \mathrm{cm}^{2}, 10$ seg por ponto, no modo contato. Paciente na fase de crosta, já não apresentava dor e nem queimação (Fig.1E). Em virtude da lesão ser relativamente extensa, foi feita a FBM também após $48 \mathrm{~h}$ para acelerar a reparação (Fig.1F). Paciente retornou para consulta de acompanhamento após 2 semanas do início do tratamento (Fig.1G), com melhora significativa da lesão.

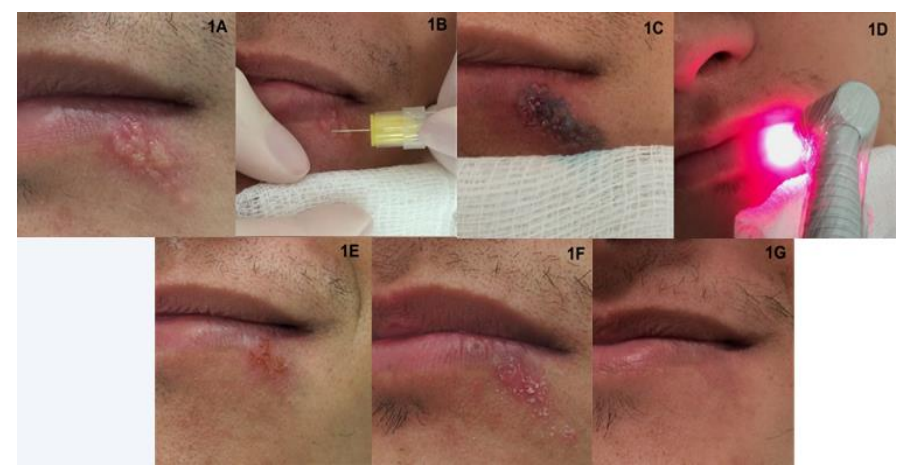

Figura 1. Aspecto inicial da fase vesicular do herpes simples labial (A). Perfuração das vesículas com agulha estéril (B). Fotossensibilizador azul de metileno $0,005 \%$ aplicado sobre a lesão (C). Irradiação com o laser diodo de baixa intensidade 660nm (D). Aspecto da lesão após 24h (E). 48h (F). 2 semanas (G).

\section{Caso Clínico 2}

Paciente I.R.S., sexo feminino, 35 anos, fototipo de pele parda, apresentou lesão de herpes na fase vesicular no lábio inferior direito (Fig.2A). A vesícula foi cuidadosamente perfurada com agulha estéril (Fig.2B) e o fluido drenado cuidadosamente com auxílio de gaze estéril para evitar espalhar infecção para área adjacente. Em seguida, foi aplicado o fotossensibilizador azul de metileno 0,005\% (Chimiolux®) sobre a lesão (Fig.2C) e, após $5 \mathrm{~min}$, o excesso de fotossensibilizador foi removido. A área da lesão foi irradiada em 2 pontos distintos com um equipamento de laser de diodo, emissão contínua na faixa do espectro da luz vermelha, em baixa intensidade com comprimento de onda de 660 nm (Twin Laser; MM Optics $\left.{ }^{\circledR}\right)$, potência de saída de 40 $\mathrm{mw}$, densidade de energia de $120 \mathrm{~J} / \mathrm{cm}^{2}, 2$ min por ponto, no modo contato (Fig.2D). Após 24 h, o paciente iniciou a terapia de fotobiomodulação (FBM). A área da lesão foi irradiada em 3 pontos, com o mesmo equipamento de laser de baixa potência $(660 \mathrm{~nm})$, porém com potência de saída de $15 \mathrm{mw}$, densidade de energia de $3,7 \mathrm{~J} / \mathrm{cm}^{2}$, $10 \mathrm{seg}$ por ponto, no modo contato. Paciente na fase de crosta (Fig.2E) relatou que sentiu coceira à noite após a TFD. Paciente retornou para consulta de acompanhamento após 1 semana do início do tratamento (Fig.2F).

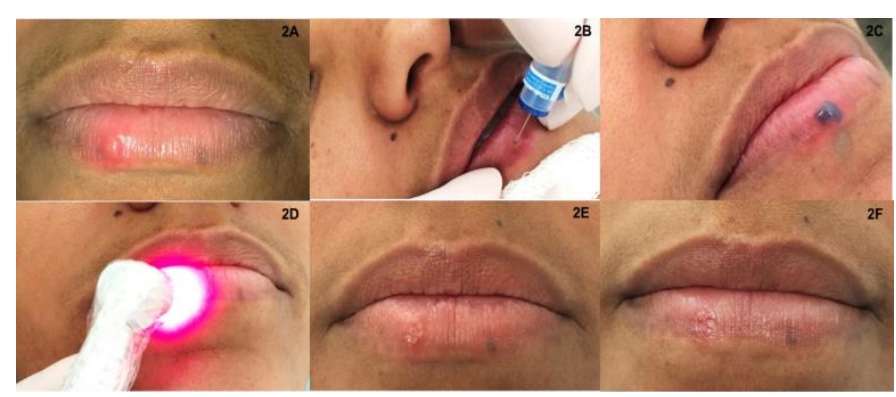

Figura 2. Aspecto inicial da fase vesicular do herpes simples labial (A). Perfuração das vesículas com agulha estéril (B). Fotossensibilizador azul de metileno $0,005 \%$ aplicado sobre a lesão (C). Irradiação com o laser diodo de baixa potência $660 \mathrm{~nm}$ (D). Aspecto da lesão após 24h (E) e 1 semana (F). 


\section{Discussão}

O protocolo de tratamento do herpes simples labial com a Terapia Fotodinâmica (TFD) foi efetivo, não teve efeitos colaterais e o processo de cicatrização foi rápido e satisfatório. A TFD promoveu inativação do vírus pela interação do fotosensibilizador (azul de metileno) e a luz (laser de baixa potência em $660 \mathrm{~nm}$ ) no tecido. O efeito antiviral da TFD depende da concentração e do tipo de fotossensibilizador, da fonte de luz e do substrato. Os parâmetros de irradiação também são importantes para se estabelecer um protocolo confiável de $\mathrm{TFD}^{3}$.

A literatura tem apontado a eficácia da TFD no tratamento do herpes simples labial ${ }^{3,4,-9}$. É uma técnica indolor e confortável, o que garante grande aceitabilidade pelos pacientes, além de não ter o inconveniente da frequência de aplicação, de 5 ou 6 vezes ao dia, como os cremes tópicos antivirais (tratamento convencional). Para o clínico, é uma técnica de baixa complexidade, de fácil execução e de resultados satisfatórios em poucos dias. Um ponto a ser destacado é a divergência de protocolos de tratamento para herpes simples labial quanto aos parâmetros do laser em si, tanto para a TFD como para a terapia de fotobiomodulação (FBM) no período de reparo da lesão, e dos benefícios da associação ou não das duas terapias; esses fatos dificultam a comparação entre estudos. ${ }^{10-12} \mathrm{O}$ que se sabe é que nenhum tratamento a laser é capaz de eliminar completamente o vírus e a sua recorrência. Entretanto, diminui a dor e o intervalo de recorrência, sem causar qualquer efeito secundário, e a TFD reduz a carga viral na fase de vesícula do herpes ${ }^{3,5}$.

Nos casos clínicos apresentados, observou-se que em 24 h após a TFD a lesão passou da fase de vesícula para a fase de crosta e com alívio de sinais e sintomas da infecção. A FBM é indicada na fase de crosta, podendo ser realizada diariamente até a eliminação completa da lesão. Os estudos de Marotti et al. (2009), Marotti et al. (2010) e Ramalho et al. (2015) apresentaram resultados clínicos também eficazes e satisfatórios utilizando protocolo de tratamento similar aos casos clínicos apresentados, diferindo quanto à concentração do fotossensibilizador e à potência do laser. Marotti et al. 2009 e 2010 utilizaram fotossensibilizadores mais concentrados, de $0,01 \%$, enquanto que no presente trabalho foi utilizada a concentração de $0,005 \%$; já Mariotti et al. 2010 e Ramalho et al. 2015 utilizaram uma potência de $100 \mathrm{~mW}$ para TFD, enquanto nesse estudo foi considerada uma potência de $40 \mathrm{~mW}$. Os achados na literatura corroboram com os resultados clínicos apresentados, mesmo que o protocolo em estudo tenha utilizado uma menor concentração do fotossensibilizador e menor potência do laser. Outro aspecto importante do protocolo clínico apresentado e dos demais estudos ${ }^{6-8}$ é que realizaram a drenagem do fluido das vesículas da lesão do herpes simples labial antes da TFD e respeitaram o tempo de 5 min de aplicação do fotossensibilizador de azul de metileno (pré-irradiação).

A TFD pode ser considerada uma alternativa de tratamento promissora para o herpes simples labial. Mostra-se eficaz, sem efeitos colaterais e mantém os tecidos saudáveis, além de um processo de cicatrização rápido e satisfatório.

\section{Conclusão}

O protocolo de tratamento do herpes simples labial, na fase vesicular com a Terapia Fotodinâmica (TFD) associada à Fotobiomodulação (FBM), foi efetivo e seguro resultando em cicatrização da área da lesão afetada, além da melhora dos sinais e sintomas do herpes simples labial, e consequente melhora no desconforto do paciente.

\section{REFERÊNCIAS}

1. Dréno B, Malkin JE, Saiag P, Batuji-Garin S. Patients' profile, burden of disease and quality of life in recurrent herpes labialis: a survey conducted in USA and France. $J$ Eur Acad Dermatol Venereol. 2012 Nov; 26(11):1458-60.

2. Chen F, Xu H, Liu J, Cui Y, Luo X, Zhou Y, Chen Q, Jiang L. Efficacy and safety of nucleoside antiviral drugs for treatment of recurrent herpes labialis: a systematic review and meta-analysis. J Oral Pathol Med. 2017 Sep; 46(8): 561-8.

3. de Paula Eduardo C1, Aranha AC, Simões A, Bello-Silva MS, Ramalho KM, Esteves-Oliveira M, de Freitas PM, Marotti J, Tunér J. Laser treatment of recurrent herpes 
labialis: a literature review. Lasers Med Sci. 2014 Jul; 29(4):1517-29.

4. Kharkwal GB, Sharma SK, Huang YY, Dai T, Hamblin MR. Photodynamic therapy for infections: clinical applications. Lasers Surg Med. 2011 Sep; 43(7):755-67.

5. Sergio LPS, Marciano RS, Teixeira GR, Canuto KL, Polignano GAC, Guimarães OR et al. Therapeutic lowintensity red laser for herpes labialis on plasmid survival and bacterial transformation. Photochem Photobiol Sci. 2013; 12:930-5.

6. Marotti J1, Aranha AC, Eduardo C de P, Ribeiro MS. Photodynamic therapy can be effective as a treatment for herpes simplex labialis. Photomed Laser Surg. 2009 Apr; 27(2):357-63.

7. Marotti J, Sperandio FF, Fregnani ER, Aranha ACR, Freitas PM, Eduardo CP. High-Intensity Laser and Photodynamic Therapy as a Treatment for Recurrent Herpes Labialis. Photomed Laser Surg. 2010; 28(3):43944.

8. Ramalho KM, Rocha RG, Corre-Aranha AC, Cunha SR, Simões A, Campos L, Eduardo C de P. Treatment of herpes simplex labialis in macule and vesicle phases with photodynamic therapy. Report of two cases. Photodiagnosis Photodyn Ther. 2015 Jun; 12(2):321-3.

9. Oniszczuka A, Kuleszaa KAW, Oniszczukb T, Kasprzaka K. The potential of photodynamic therapy (PDT). Experimental investigations and clinical use. Biomed Pharmacother. 2016 Oct; 83: 912-29.

10. Dougal G, Lee SY. Evaluation of the efficacy of low-level light therapy using $1072 \mathrm{~nm}$ infrared light for the treatment of herpes simplex labialis. Clin Exp Dermatol. 2013 Oct; 38(7):713-8.

11. Honarmand M, Farhadmollashahi L, Vosoughirahbari E. Comparing the effect of diode laser against acyclovir cream for the treatment of herpes labialis. J Clin Exp Dent. 2017; 9(6):729-32.

12. Brignardello-Petersen R. Treatment of lesions associated with herpes labialis with low level laser therapy may result in a decrease of pain and recovery time compared with acyclovir. J Am Dent Assoc. 2017 Oct;148(10):e153. 